\title{
THE INDIRECT BOUNDARY INTEGRAL METHOD FOR CURVED CRACKS IN PLANE ELASTICITY
}

\author{
BEONG-IN YUN
}

\begin{abstract}
For curved crack problems in plane elasticity, subjected to the traction conditions on the crack faces, we present a system of boundary integral equations. The procedure is based on the indirect boundary integral method in terms of real variables. For efficient mathernatical analysis, we decompose the singular kernel into the Cauchy singular part and the regular one. As a result, solvability of the presented system is proved and availability of the present approach is shown by the numerical example of a circular arc crack.
\end{abstract}

\section{Introduction}

We consider the linear elastic field in the infinite plane, with body force terms omitted, which contains a curved crack, $\Gamma$ subjected to the equal and opposite loading on the crack faces. Since $\Sigma t_{i}=t_{i}^{+}+t_{i}^{-}=$ $0(i=1,2)$ for the tractions $t_{i}^{+}$and $t_{i}^{-}$on the upper - and lower - crack faces, respectively, the traditional direct boundary integral formulation [1] gives

(1) $\quad u_{i}(x)=\left\{\begin{array}{l}-\int_{\Gamma} T_{i j}(x, y) \Delta u_{j}(y) d \Gamma(y), \quad x \in \mathbb{R}^{2} \backslash \Gamma, \\ \frac{1}{2} \Delta u_{i}(x)-\int_{\Gamma} T_{i j}(x, y) \Delta u_{j}(y) d \Gamma(y), \quad x \in \Gamma,\end{array}\right.$

Received March 19, 2002. Revised June 7, 2002.

2000 Mathematics Subject Classification: 45F15, $65 \mathrm{H} 10$.

Key words and phrases: boundary integral method, singular integral, curved crack.

This work was supported by Korea Research Foundation Grant (KRF-2000-015DP0042). 
where $T_{i j}$ 's are the Kelvin's fundamental solutions for tractions, $u_{i}$ 's are the displacements and $\Delta u_{i}=u_{i}^{+}-u_{i}^{-}$are the crack opening displacements [5]. It is well known that these equations have serious deficiencies:

1. There are two unknowns, $u_{i}$ and $\Delta u_{i}$.

2. Any set of equal and opposite tractions, say, $\Sigma t_{i}=0$ will result in the same equation (1).

3. The stresses (or tractions) induced by the formula (1) have hyper singular integrals.

In order to overcome these problems many works such as subregion method [2], crack Green's function technique, dislocation theory $[6,10]$ and complex variable method [3] have been presented. However it is known that these methods are too restrictive to the general crack geometry and have the drawback that their essential ingredients must be constructed in full for every individual situation.

Recently, another remarkable technique, so called dual boundary element method (DBEM) was developed by Portela [8] and Portela at al [9]. In this method the displacement boundary integral equation is applied to one of the crack faces and the traction boundary integral equation on the other, for which there are no mathematical analysis on the solvability. On the other hand, for curved crack problems, Wendland and Stephan [11] have studied on the hypersingular integral equations obtained by applying the traction operator to the direct boundary integral equations. They gave complete results on the solvability of the equations and on the regularity of the solutions in Sobolev spaces. However, in this case, one cannot help manipulating hypersingular integrals in numerical scheme.

In this paper we introduce the indirect boundary integral method for curved crack problems which is based on the displacement representation in the form of the single-layer potential. This results in a system of two integral equations of real variables which contain Cauchy singular kernels. The integral operators corresponding to these kernels are decomposed for efficient mathematical analysis and numerical evaluation. We expect this approach can avoid the difficulties of direct boundary integral methods. Moreover, as a main result of this paper, solvability of the system of induced integral equations is proved.

In the next section we formulate a system of boundary integral equations on the curved crack face and, in Section 3, prove the solvability of the boundary integral equations. Section 4 shows efficiency of the present method by a numerical example of a circular arc crack. 


\section{Indirect boundary integral method}

In the isotropic, homogeneous and linear elastic field without body forces, the displacement vector $\mathbf{u}=\left(u_{1}, u_{2}\right)$ satisfies

$$
\mu \nabla^{2} \mathbf{u}+(\lambda+\mu) \operatorname{grad} \cdot \operatorname{div} \mathbf{u}=0 \quad \text { in } \quad \Omega=\mathbb{R}^{2} \backslash \Gamma,
$$

where $\mu=E /(2(1+\nu)), \lambda=2 \mu \nu /(1-2 \nu) . \nu$ is the Poisson ratio and $E$ is the Young's modulus. In this paper we consider the state of the plane strain. Under the assumption that the equal and opposite loading is applied on the crack faces, we identify $\Gamma$ with the upper crack face and denote $\mathbf{n}_{P}=\left(n_{1}(P), n_{2}(P)\right)$ as a outward normal vector at $P \in \Gamma$. Then, for the tractions $t_{i}(i=1,2)$ defined by

$$
t_{i}(P)=\sum_{j=1}^{2} \sigma_{i j}(P) n_{j}(P), \quad P \in \Gamma,
$$

we consider the boundary conditions

$$
\left\{\begin{array}{l}
t_{1}(P)=f(P), \\
t_{2}(P)=g(P), \quad P \in \Gamma,
\end{array}\right.
$$

where $f(P)$ and $g(P)$ are given.

\subsection{Derivation of the boundary integral equations}

As mentioned in the previous section the traditional direct boundary integral formulae result in the equation (1) which contains some mathematical difficulties. To avoid these problems, we suggest the indirect boundary integral formulae of the displacements $u_{i}$ such as

$$
u_{i}(P)=\int_{\Gamma} U_{i j}(P, Q) \lambda_{j}(Q) d \Gamma(Q), \quad P \in \mathbb{R}^{2},
$$

in which rigid body motions are ignored. In (4) $U_{i j}(P, Q)$ 's are the Kelvin's fundamental solutions for displacements [1] and $\lambda_{i}(P)$ 's are the density functions to be determined by the condition (3) after translation of the formula (4) into the traction formulae. Since, in the equation (4), $u_{i}(P)=\log |P| \int_{\Gamma} \lambda_{i}(Q) d \Gamma(Q)+O(1)$ as $|P| \rightarrow \infty$, we assume the boundedness of the displacements at infinity which is satisfied by the conditions

$$
\int_{\Gamma} \lambda_{i}(Q) d \Gamma(Q)=0, \quad i=1,2
$$


On the other hand, from the equation (4), the stresses become

$$
\sigma_{i j}\left(P^{\prime}\right)=\sum_{k=1}^{2} \int_{\Gamma} D_{i j k}\left(P^{\prime}, Q\right) \lambda_{k}(Q) d \Gamma(Q), \quad P^{\prime} \in \Omega,
$$

where, for two points $P^{\prime}=\left(x_{1}, x_{2}\right)$ and $Q=\left(\xi_{1}, \xi_{2}\right)$ in $\Omega$ with $r=$ $\left|Q-P^{\prime}\right|$, and for a constant $A$ denoted as

$$
A=\frac{1}{4 \pi(1-\nu)},
$$

the kernel $D_{i j k}$ is defined by

$$
\begin{aligned}
D_{i j k}\left(P^{\prime}, Q\right) & =\frac{2 \mu \nu}{1-2 \nu}\left\{\frac{\partial U_{1 k}}{\partial x_{1}}+\frac{\partial U_{2 k}}{\partial x_{2}}\right\} \delta_{i j}+\frac{\mu}{2}\left\{\frac{\partial U_{i k}}{\partial x_{j}}+\frac{\partial U_{j k}}{\partial x_{i}}\right\} \\
& =\frac{A}{r}\left\{(1-2 \nu)\left(r_{, k} \delta_{i j}+r_{, j} \delta_{k i}-r_{, i} \delta_{j k}\right)+2 r_{, i} r_{, j} r_{, k}\right\}
\end{aligned}
$$

For a fixed point $P \in \Gamma$, let $L_{P}$ be the line through $P$ parallel to the normal vector $\mathbf{n}_{P}=\left(n_{1}(P), n_{2}(P)\right)$. Multiplying both sides of (6) by $\mathbf{n}_{P}$ and observing that

$$
r_{, j}:=\frac{\partial r}{\partial \xi_{j}}=-\frac{\partial r}{\partial x_{j}}, \quad \sum_{j=1}^{2} r_{, j} n_{j}(P)=-\frac{\partial r}{\partial \mathbf{n}_{P}},
$$

we define a function $\bar{t}_{i}$ in the region $L_{P} \cap \Omega$ such as

$$
\begin{aligned}
\bar{t}_{i}\left(P^{\prime}\right) & =\sum_{j=1}^{2} \sigma_{i j}\left(P^{\prime}\right) n_{j}(P) \\
& =\sum_{k=1}^{2} \int_{\Gamma} T_{i k}^{*}\left(P^{\prime}, Q\right) \lambda_{k}(Q) d \Gamma(Q), \quad P^{\prime} \in L_{P} \cap \Omega
\end{aligned}
$$

where

$$
\begin{aligned}
& T_{i k}^{*}\left(P^{\prime}, Q\right)= \sum_{j=1}^{2} D_{i j k}\left(P^{\prime}, Q\right) n_{j}(P) \\
&=-\frac{A}{r}\left\{(1-2 \nu)\left(r_{, k} n_{i}(P)-r_{, i} n_{k}(P)\right)\right. \\
&\left.+\left[(1-2 \nu) \delta_{i k}+2 r_{, i} r_{, k}\right] \frac{\partial r}{\partial \mathbf{n}_{P}}\right\} .
\end{aligned}
$$


For $i \neq k$, this can be rewritten by

$$
\begin{aligned}
& T_{i i}^{*}=-\frac{A}{r}\left\{\left[(1-2 \nu)+2\left(r_{, i}\right)^{2}\right] \frac{\partial r}{\partial \mathbf{n}_{P}}\right\}, \\
& T_{i k}^{*}=-\frac{A}{r}\left\{(1-2 \nu)\left(r_{, k} n_{i}(P)-r_{, i} n_{k}(P)\right)+2 r_{, i} r_{, k} \frac{\partial r}{\partial \mathbf{n}_{P}}\right\} .
\end{aligned}
$$

Then, by the limiting process $P^{\prime} \rightarrow P \in \Gamma$, we have the well known result of the boundary integral equation for the boundary points as follows:

Lemma 1. For any twice continuously differentiable boundary curve $\Gamma$, the function $\bar{t}_{i}\left(P^{\prime}\right)$ defined in (8) satisfies the following jump relation.

$$
\lim _{p^{\prime} \rightarrow p} \bar{t}_{i}\left(P^{\prime}\right)=\frac{1}{2} \lambda_{i}(P)+\sum_{k=1}^{2} f_{\Gamma} T_{i k}^{*}(P, Q) \lambda_{k}(Q) d \Gamma(Q), P \in \Gamma
$$

for $i=1,2$.

Identifying the limit value of $\bar{t}_{i}\left(P^{\prime}\right)$ in (11) with the traction $t_{i}(P)$, from the boundary conditions (3), we have the boundary integral equations as

$$
\begin{aligned}
& \left(\frac{1}{2} I+S_{11}\right) \lambda_{1}(P)+\left(S_{12} \lambda_{2}\right)(P)=f(P), \\
& \left(S_{21} \lambda_{1}\right)(P)+\left(\frac{1}{2} I+S_{22}\right) \lambda_{2}(P)=g(P), \quad P \in \Gamma,
\end{aligned}
$$

where $S_{i k}$ are integral operators defined by

$$
\left(S_{i k} \phi\right)(P)=f_{\Gamma} T_{i k}^{*}(P, Q) \phi(Q) d \Gamma(Q), \quad i, k=1,2 .
$$

In the right hand side of this formula, the symbol of the integral means the Cauchy principal value when $i \neq k$. The system of integral equations in (12) can be written by the matrix form

$$
M \widetilde{x}=\widetilde{y},
$$

where $\tilde{x}=\left(\lambda_{1}, \lambda_{2}\right)^{T}, \widetilde{y}=(f, g)^{T}$ and $M$ is the matrix operator denoted by

$$
M=\left[\begin{array}{cc}
\frac{1}{2} I+S_{11} & S_{12} \\
S_{21} & \frac{1}{2} I+S_{22}
\end{array}\right]
$$




\subsection{Decomposition of the kernels}

We decompose the integral operator $S_{12}$ (and $S_{21}$ ) into the Cauchy singular operator and a regular one in order to prove solvability of the equation (14) efficiently. Firstly we assume that the boundary curve $\Gamma$ is parametrized by

$P=\beta(x)=(x, \phi(x)) \quad$ and $\quad Q=\beta(\xi)=(\xi, \phi(\xi)) \quad(-1 \leq x, \xi \leq 1)$, where $\phi(x) \in C^{2}[-1,1]$. Then, for $N(x)=\sqrt{1+\phi^{\prime}(x)^{2}}=\left|\beta^{\prime}(x)\right|$, we have

$$
\begin{aligned}
\mathbf{n}_{p} & =\left(n_{1}, n_{2}\right)=\left(-\phi^{\prime}(x), 1\right) / N(x), \\
r^{2} & =|Q-P|^{2} \\
& =(\xi-x)^{2}\left\{1+\left[\frac{\phi(\xi)-\phi(x)}{\xi-x}\right]^{2}\right\}:=(\xi-x)^{2} m(x, \xi), \\
\left(r_{, 1}\right)^{2} & =\frac{1}{m(x, \xi)}, \quad\left(r_{, 2}\right)^{2}=1-\frac{1}{m(x, \xi)}, \\
r_{, 1} r_{, 2} & =\left[\frac{\phi(\xi)-\phi(x)}{\xi-x}\right] \frac{1}{m(x, \xi)},
\end{aligned}
$$

and

$$
\begin{aligned}
\frac{\partial \log r}{\partial \mathbf{n}_{P}} & =\frac{(\phi(\xi)-\phi(x))-(\xi-x) \phi^{\prime}(x)}{(\xi-x)^{2} m(x, \xi) N(x)} \\
\left(\frac{1}{r}\right) r_{, 2} n_{1} & =\frac{-1}{N(x) m(x, \xi)(\xi-x)}\left[\frac{\phi(\xi)-\phi(x)}{\xi-x}\right] \phi^{\prime}(x), \\
\left(\frac{1}{r}\right) r_{, 1} n_{2} & =\frac{1}{N(x) m(x, \xi)(\xi-x)} .
\end{aligned}
$$

Furthermore, by denoting as

$$
\Phi(x, \xi)=\frac{\phi(\xi)-\phi(x)-(\xi-x) \phi^{\prime}(x)}{(\xi-x)^{2}}
$$

we have

$$
\begin{aligned}
& \frac{1}{r}\left(r_{, 2} n_{1}-r_{, 1} n_{2}\right) \\
& \quad=\frac{-1}{N(x) m(x, \xi)(\xi-x)}\left\{\left[\frac{\phi(\xi)-\phi(x)}{\xi-x}\right] \phi^{\prime}(x)+1\right\} \\
& \quad=\frac{-1}{N(x)}\left\{\frac{1}{\xi-x}-\frac{1}{m(x, \xi)}\left[\frac{\phi(\xi)-\phi(x)}{\xi-x}\right] \Phi(x, \xi)\right\} .
\end{aligned}
$$


The last equality holds by the fact that

$$
\begin{aligned}
& {\left[\frac{\phi(\xi)-\phi(x)}{\xi-x}\right] \phi^{\prime}(x)+1} \\
& =\left[\frac{\phi(\xi)-\phi(x)}{\xi-x}\right] \phi^{\prime}(x)+m(x, \xi)-\left[\frac{\phi(\xi)-\phi(x)}{\xi-x}\right]^{2} \\
& =m(x, \xi)-\left[\frac{\phi(\xi)-\phi(x)}{\xi-x}\right] \frac{1}{\xi-x}\left\{\phi(\xi)-\phi(x)-(\xi-x) \phi^{\prime}(x)\right\} .
\end{aligned}
$$

By the results in (16)-(19), the kernels $T_{i j}^{*}=T_{i j}^{*}(\beta(x), \beta(\xi))$ can be rewritten as

$$
\begin{aligned}
N(x) T_{11}^{*}= & -\frac{1}{m(x, \xi)}\left[c+\frac{2 A}{m(x, \xi)}\right] \Phi(x, \xi), \\
N(x) T_{22}^{*}= & -\frac{1}{m(x, \xi)}\left[c+2 A\left(1-\frac{1}{m(x, \xi)}\right)\right] \Phi(x, \xi), \\
N(x) T_{12}^{*}= & \frac{c}{\xi-x} \\
& -\left(c+\frac{2 A}{m(x, \xi)}\right) \frac{1}{m(x, \xi)}\left[\frac{\phi(\xi)-\phi(x)}{\xi-x}\right] \Phi(x, \xi) \\
:= & \frac{c}{\xi-x}+h_{12}(x, \xi), \\
N(x) T_{21}^{*}= & -\frac{c}{\xi-x} \\
& +\left(c-\frac{2 A}{m(x, \xi)}\right) \frac{1}{m(x, \xi)}\left[\frac{\phi(\xi)-\phi(x)}{\xi-x}\right] \Phi(x, \xi) \\
:= & -\frac{c}{\xi-x}+h_{21}(x, \xi),
\end{aligned}
$$

where the constant $c$ is defined by

$$
c=A(1-2 \nu)=\frac{1-2 \nu}{4 \pi(1-\nu)} .
$$

It should be noted that the functions $h_{12}$ and $h_{21}$ in (20) are continuous functions on the region $[-1,1] \times[-1,1]$.

For the convenience of the formulation we multiply both sides of the equations in (12) by $N(x)=\left|\beta^{\prime}(x)\right|$ after parametrization of the variables, and then denote the unknown density function as $\mu_{i}(x)=$ $\lambda_{i}(\beta(x))\left|\beta^{\prime}(x)\right|$. Then the integral operators $S_{i j}$ are reset as those multiplied by $N(x)$. Moreover we decompose the singular integral operators 
$S_{12}$ and $S_{21}$ as

$$
\begin{aligned}
S_{12} \mu_{2}(x) & =c \int_{-1}^{1} \frac{1}{\xi-x} \mu_{2}(\xi) d \xi+\int_{-1}^{1} h_{12}(x, \xi) \mu_{2}(\xi) d \xi \\
& :=S_{12}^{\prime} \mu_{2}(x)+S_{12}^{\circ} \mu_{2}(x)
\end{aligned}
$$

and

$$
\begin{aligned}
S_{21} \mu_{1}(x) & =-c \int_{-1}^{1} \frac{1}{\xi-x} \mu_{1}(\xi) d \xi+\int_{-1}^{1} h_{21}(x, \xi) \mu_{1}(\xi) d \xi \\
& :=S_{21}^{\prime} \mu_{1}(x)+S_{21}^{\circ} \mu_{1}(x) .
\end{aligned}
$$

Then the equation (12) or (14) becomes, for $-1 \leq x \leq 1$,

$$
\left[\begin{array}{cc}
\frac{1}{2} I+S_{11} & \left(S_{12}^{\prime}+S_{12}^{\circ}\right) \\
\left(S_{21}^{\prime}+S_{21}^{\circ}\right) & \frac{1}{2} I+S_{22}
\end{array}\right]\left[\begin{array}{l}
\mu_{1}(x) \\
\mu_{2}(x)
\end{array}\right]=N(x) \cdot\left[\begin{array}{l}
f(\beta(x)) \\
g(\beta(x))
\end{array}\right] .
$$

\section{Solvability of the indirect boundary integral equations}

For a closed curve, the indirect boundary integral method of real variables has been rigorously investigated by means of the mathematical structure of argument [4]. However, as par as the author is aware, there are no account of the solvability of the indirect integral equations in the case of an open arc or crack. It is notable that Wendland and Stephan [11] gave complete results on the solvability of hypersingular integral equations, based on the direct boundary integral method, for crack problems. In this section we study on the functional properties of the integral operators introduced in the previous section and then prove solvability of the system of boundary integral equations (23).

Theorem 2. Assume that the boundary curve $\Gamma$ is a twice continuously differentiable and convex or concave open arc with $\frac{\pi}{2} \leq \theta_{P} \leq \pi$ for all $P \in \Gamma$, where $\theta_{P}$ is an interior angle on $P$ between two end points of $\Gamma$. Then the operators $\frac{1}{2} I+S_{11}$ and $\frac{1}{2} I+S_{22}$ are invertible on the space $C(\Gamma)$ for any Poisson ratio $0<\nu<\frac{1}{2}$.

Proof. We show that the adjoint of $\frac{1}{2} I+S_{i i}$, namely, $\frac{1}{2} I+\tilde{S}_{i i}$ is invertible. $\tilde{S}_{i i}$ is defined by

$$
\left(\tilde{S}_{i i} \phi\right)(P)=\int_{\Gamma} T_{i i}(P, Q) \phi(Q) d \Gamma(Q)
$$


where

$$
T_{i i}(P, Q)=-A\left[(1-2 \nu)+2\left(r_{, i}\right)^{2}\right] \frac{\partial \log r}{\partial \mathbf{n}_{Q}}, \quad r=|Q-P| .
$$

Since the kernel $T_{i i}(i=1,2)$ is continuous on $\Gamma$, the operator $\tilde{S}_{i i}$ is compact. Referring to the Fredholm alternative, it is sufficient to show that $\frac{1}{2} I+\tilde{S}_{i i}$ is injective.

Assume that $\lambda_{i}$ in $C(\Gamma)$ is a non-trivial solution of the homogeneous equation

$$
\left(\frac{1}{2} I+\tilde{S}_{i i}\right) \lambda_{i}(P)=0
$$

Then

$$
\begin{aligned}
\lambda_{i}(P) & =-2 \int_{\Gamma} T_{i i}(P, Q) \lambda_{i}(Q) d \Gamma(Q) \\
& =-2 \int_{\Gamma} A\left[(1-2 \nu)+2\left(r_{, i}\right)^{2}\right]\left(\frac{\partial}{\partial \mathbf{n}_{Q}} \log r\right) \lambda_{i}(Q) d \Gamma(Q) .
\end{aligned}
$$

Since $r_{, i} \leq 1$ and $1-2 \nu>0$ for all $\nu<0.5$,

$$
0<A\left[(1-2 \nu)+2\left(r_{, i}\right)^{2}\right]<\frac{1}{4 \pi(1-\nu)}[(1-2 \nu)+2+(1-2 \nu)]=\frac{1}{\pi}
$$

and, in Yun and Lee [13], it has been proved that

$$
\int_{\Gamma} \frac{\partial}{\partial \mathbf{n}_{Q}} \log r d \Gamma(Q)=\theta_{P}-\pi
$$

Moreover the sign of $\frac{\partial}{\partial n_{Q}} \log r$ is not changed because the boundary $\Gamma$ has been assumed to be convex or concave. This results in

$$
\begin{aligned}
\int_{\Gamma}\left|\frac{\partial}{\partial \mathbf{n}_{Q}} \log r\right| d \Gamma(Q) & =\left|\int_{\Gamma} \frac{\partial}{\partial \mathbf{n}_{Q}} \log r d \Gamma(Q)\right| \\
& =\pi-\theta_{P} \leq \frac{\pi}{2}, \quad P \in \Gamma .
\end{aligned}
$$

Therefore it follows that

$$
\begin{aligned}
\left|\lambda_{i}(P)\right| & \leq 2\|\lambda\|_{\infty} \int_{\Gamma}\left|T_{i i}(P, Q)\right| d \Gamma(Q) \\
& <2\|\lambda\|_{\infty}\left(\frac{1}{\pi} \cdot \frac{\pi}{2}\right)=\|\lambda\|_{\infty}
\end{aligned}
$$

that is, $\left|\lambda_{i}(P)\right|<\left\|\lambda_{i}\right\|_{\infty}$ for all $P$ in $\Gamma$. This is a contradiction so that the adjoint operator $\frac{1}{2} I+\tilde{S}_{i i}$ is invertible, which completes the proof. 
If we introduce the Banach space $L_{2}(J, w)$ of all measurable functions $\phi$ on a line segment $J=[-1,1]$ with the norm $\|\phi\|_{2, w}=\|w \phi\|_{L_{2}(J)}$, where $w$ is a related weight, then we have the following lemma.

THEOREM 3. For a weight $w(x)=1 / \sqrt{1-x^{2}}(-1 \leq x \leq 1)$, the Cauchy singular integral operator

$$
S_{12}^{\prime}: L_{2}\left([-1,1], w^{-\frac{1}{2}}\right) \longrightarrow L_{2}\left([-1,1], w^{-\frac{1}{2}}\right)
$$

is a bounded linear operator with $\left\|S_{12}^{\prime}\right\| \leq \pi c$ and $I+4 S_{12}^{\prime}{ }^{2}$ is invertible.

Proof. For any function $\phi(x) \in L_{2}\left([-1,1], w^{-\frac{1}{2}}\right)$, referring the fact that $C[-1,1]$ is a dense subset of $L_{2}\left([-1,1], w^{\alpha}\right), \alpha<1$, we may take a continuous function $f(x)$ such that $w^{\frac{1}{2}} f \in C[-1,1]$ and $\| w^{-\frac{1}{2}} \phi-$ $w^{\frac{1}{2}} f \|_{L_{2}[-1,1]}<\epsilon$ for sufficiently small $\epsilon$.

Then $\phi(x)$ can be approximated by the function $\lambda(x)=w(x) f(x)$ $\in L_{2}\left([-1,1], w^{-\frac{1}{2}}\right)$ as

$$
\begin{aligned}
\|\phi-\lambda\|_{2, w^{-\frac{1}{2}}} & =\left\|w^{-\frac{1}{2}}(\phi-w f)\right\|_{L_{2}[-1,1]} \\
& =\left\|w^{-\frac{1}{2}} \phi-w^{\frac{1}{2}} f\right\|_{L_{2}[-1,1]}<\epsilon .
\end{aligned}
$$

Since the continuous function $f$ can be represented by the Chebyshev polynomial expansion, we set

$$
\lambda(x)=w(x) \sum_{n \geq 0} a_{n} T_{n}(x) .
$$

By using the orthogonality of the first kind Chebyshev polynomials $\left\{T_{n}\right\}$ with the weight $w(x)$, we have

$$
\begin{aligned}
\|\lambda\|_{2, w^{-\frac{1}{2}}}^{2} & =\left\|w(x) \sum_{n \geq 0} a_{n} T_{n}(x)\right\|_{2, w^{-\frac{1}{2}}}^{2} \\
& =\int_{-1}^{1} w(x)\left[\sum_{n \geq 0} a_{n} T_{n}(x)\right]^{2} d x \\
& =\sum_{n \geq 0} a_{n}{ }^{2} \int_{-1}^{1} w(x) T_{n}(x)^{2} d x \\
& =\left(\frac{\pi}{2}\right)\left\{2 a_{0}^{2}+\sum_{n \geq 1}{a_{n}}^{2}\right\}<\infty .
\end{aligned}
$$


On the other hand, the result in Mikhlin and Prössdorf [7] such as

$$
f_{-1}^{1} \frac{w(\xi)}{\xi-x} T_{n}(\xi) d \xi= \begin{cases}0, & n=0 \\ \pi U_{n-1}(x), & n \geq 1\end{cases}
$$

and the orthogonality of the second kind Chebyshev polynomials, $\left\{U_{n}\right\}$ with the weight $w^{-1}(x)$ imply

$$
\begin{aligned}
\left\|S_{12}^{\prime} \lambda\right\|_{2, w^{-\frac{1}{2}}}^{2} & =c^{2}\left\|\sum_{n \geq 0} a_{n} f_{-1}^{1} \frac{w(\xi)}{\xi-x} T_{n}(\xi) d \xi\right\|_{2, w^{-\frac{1}{2}}}^{2} \\
& =(\pi c)^{2}\left\|\sum_{n \geq 1} a_{n} U_{n-1}(x)\right\|_{2, w^{-\frac{1}{2}}}^{2} \\
& =(\pi c)^{2} \int_{-1}^{1}\left(\sum_{n \geq 1} a_{n} U_{n-1}(x)\right)^{2} w^{-1}(x) d x \\
& =(\pi c)^{2} \sum_{n \geq 1} a_{n}^{2} \int_{-1}^{1} U_{n-1}(x)^{2} w^{-1}(x) d x \\
& =(\pi c)^{2}\left(\frac{\pi}{2}\right) \sum_{n \geq 1} a_{n}^{2}<(\pi c)^{2}\|\lambda\|_{2, w^{-\frac{1}{2}}}^{2}
\end{aligned}
$$

where $c$ is the constant given in (20). Thus $S_{12}^{\prime}$ is a bounded operator with $\left\|S_{12}^{\prime}\right\| \leq \pi c$. Moreover by observing

$$
\pi c=\frac{1-2 \nu}{4(1-\nu)}<\frac{1}{4} \quad \text { for all } \quad 0<\nu<\frac{1}{2}
$$

we have

$$
\left\|4 S_{12}^{\prime 2}\right\| \leq 4\left\|S_{12}^{\prime}\right\|^{2} \leq 4(\pi c)^{2}<\frac{1}{4}
$$

which implies that $I+4{S_{12}^{\prime}}^{2}$ is invertible.

In fact it is known that the operator $S_{12}^{\prime}$ is the invertible continuous operator from the space $L_{2}\left([-1,1], w^{-\frac{1}{2}}\right)$ onto itself [7]. For the solvability of the system given in (23), we define a space $X=L_{2}\left([-1,1], w^{-\frac{1}{2}}\right) \times$ $L_{2}\left([-1,1], w^{-\frac{1}{2}}\right)$ with the weight $w(x)=1 / \sqrt{1-x^{2}}$. Then we have the main theorem as follows.

THEOREM 4. The system of integral equations (23) is uniquely solvable on the space $X$. 
Proof. Noting that $S_{21}^{\prime}=-S_{12}^{\prime}$, we decompose the matrix in the left hand side of (23) such as

$$
M=\left[\begin{array}{cc}
\frac{1}{2} I & S_{12}^{\prime} \\
-S_{12}^{\prime} & \frac{1}{2} I
\end{array}\right]+\left[\begin{array}{ll}
S_{11} & S_{12}^{\circ} \\
S_{21}^{\circ} & S_{22}
\end{array}\right]=A+B
$$

Then it can be seen that $B$ is compact since the kernels of the integral operators $S_{11}, S_{22}, S_{12}^{\circ}$ and $S_{21}^{\circ}$ are continuous. From Lemma 3.2 we can see that $A$ is an invertible operator with its inverse,

$$
A^{-1}=\left[\begin{array}{cc}
2\left(I+4{S_{12}^{\prime}}^{2}\right)^{-1} & -4{S_{12}^{\prime}}^{\prime}\left(I+4{S_{12}^{\prime}}^{2}\right)^{-1} \\
4{S_{12}^{\prime}\left(I+4{S_{12}^{\prime}}^{2}\right)^{-1}}^{\prime} & 2\left(I+4{S_{12}^{\prime}}^{2}\right)^{-1}
\end{array}\right]
$$

by observing that $A A^{-1}=A^{-1} A=I$. Therefore the operator

$$
M=A\left(I+A^{-1} B\right)
$$

is invertible if it is injective. In the homogeneous equation

$$
M \mathbf{v}=\mathbf{0}, \quad \mathbf{v}=\left(v_{1}, v_{2}\right)^{T}
$$

of (23), if we interpret $\mathbf{v}$ as a vector of the crack opening displacements with traction zero and then apply the traction operator to the above homogeneous equation, the resultant equation has the trivial solution $\mathbf{v}=\mathbf{0}$ by Theorem 2.3 in Wendland and Stephan[11]. This completes the proof.

\section{A numerical example}

Firstly, referring to Ladner [6] and $\mathrm{Xu}$ and Keer [12], we represent the unknown density functions of the boundary integral equations in (23) as

$$
\mu_{1}(x)=w(x) \sum_{n=0}^{N-1} a_{n} T_{n}(x), \quad \mu_{2}(x)=w(x) \sum_{n=0}^{N-1} b_{n} T_{n}(x),
$$

where $T_{n}(x)$ is the first kind Chebyshev polynomial and $w(x)$ is the related weight function such as

$$
w(x)=\left(1-x^{2}\right)^{-\frac{1}{2}}, \quad-1 \leq x \leq 1 .
$$

It should be noted that we can avoid numerical evaluation of the Cauchy principal value integrals, $S_{12}^{\prime} \mu_{i}(x)=-S_{21}^{\prime} \mu_{i}(x)$ in (23) by the fact

$$
f_{-1}^{1} \frac{1}{\xi-x} w(\xi) T_{n}(\xi) d \xi=\pi U_{n-1}(x), \quad n \geq 0
$$


where $U_{n-1}(x)$ is the second kind Chebyshev polynomial with $U_{-1}(x)=$ 0 . However, the system of equations (23) still contains weakly singular integrals and thus the standard quadrature rule for these integrals may result in low convergence. To overcome this problem, we use a nonlinear coordinate transformation as follows:

$$
\xi=1-2 \gamma_{m}^{\operatorname{Sidi}}\left(\frac{1-t}{2}\right), \quad-1 \leq t \leq 1
$$

where $\gamma_{m}^{\text {Sidi }}$ is a well known Sidi sigmoidal transformation of order $m$ defined by

$$
\gamma_{m}^{\mathrm{Sid}}(x)=\frac{\sqrt{\pi} \Gamma((m+1) / 2)}{\Gamma(m / 2)} \int_{0}^{x}(\sin \pi \xi)^{m-1} d \xi, \quad 0 \leq x \leq 1
$$

In the work of Johnston and Elliott [14], it has been proved that the sigmoidal transformation technique like (26) sufficiently improves the errors of the numerical evaluation using the Gauss quadrature rule for weakly singular integrals.

Once the density functions, $\mu_{1}$ and $\mu_{2}$ are obtained by solving the equations in (23), we can evaluate displacements $u_{1}(x, y)$ and $u_{2}(x, y)$ for arbitrary source point, $P=(x, y) \in \mathbb{R}^{2}$. That is, from the formulae in (4), the displacements are

$$
u_{i}(x, y)=\int_{-1}^{1} U_{i j}(x, y ; \xi) \mu_{j}(\xi) d \xi, \quad i, j=1,2,
$$

where the kernels $U_{i j}(x, y ; \xi)$ take the form of

$$
\begin{aligned}
& U_{11}(x, y ; \xi)=\frac{\mathrm{A}}{2 \mu}\left\{(3-4 \nu) \log \left(\frac{1}{r}\right)+\left(\frac{x-\xi}{r}\right)^{2}\right\}, \\
& U_{12}(x, y ; \xi)=U_{21}(x, y ; \xi)=\frac{\mathrm{A}}{2 \mu} \frac{(x-\xi)(y-\phi(\xi))}{r^{2}}, \\
& U_{22}(x, y ; \xi)=\frac{\mathrm{A}}{2 \mu}\left\{(3-4 \nu) \log \left(\frac{1}{r}\right)+\left(\frac{y-\phi(\xi)}{r}\right)^{2}\right\}
\end{aligned}
$$

and $r=\sqrt{(x-\xi)^{2}+(y-\phi(\xi))^{2}}$. 
On the other hand, from the formulae in (6), we define stress functions $F(P), G(P)$ and $H(P)$ for any source point $P=(x, y)$ as

$$
\begin{aligned}
F(P) & :=\sigma_{11}(P)+\sigma_{22}(P) \\
& =\frac{1}{\pi} \int_{-1}^{1} \frac{1}{\xi-x} \cdot \frac{1}{\widetilde{m}(x, \xi)}\left\{\mu_{1}(\xi)+\left[\frac{\phi(\xi)-y}{\xi-x}\right] \mu_{2}(\xi)\right\} d \xi \\
G(P) & :=\sigma_{22}(P)-\sigma_{11}(P) \\
& =\int_{-1}^{1} \frac{1}{\xi-x} \cdot \frac{2 A}{\widetilde{m}(x, \xi)}\left(1-\frac{2}{\widetilde{m}(x, \xi)}\right) \\
& \times\left\{\mu_{1}(\xi)+\left[\frac{\phi(\xi)-y}{\xi-x}\right] \mu_{2}(\xi)\right\} d \xi \\
H(P) & \left.:=2 \sigma_{12}(P) \quad-\left[c-2 A\left(1-\frac{1}{\widetilde{m}(x, \xi)}\right)\right] \mu_{2}(\xi)\right\} d \xi \\
= & \int_{-1}^{1} \frac{1}{\xi-x} \cdot \frac{2}{\widetilde{m}(x, \xi)}\left\{\left[c+\frac{2 A}{\widetilde{m}(x, \xi)}\right]\left[\frac{\phi(\xi)-y}{\xi-x}\right] \mu_{1}(\xi)\right.
\end{aligned}
$$

in which

$$
\widetilde{m}(x, \xi)=1+\left[\frac{\phi(\xi)-y}{\xi-x}\right]^{2} .
$$

As an example we consider a circular-arc crack, $\Gamma$ parametrized by

$$
\beta(\xi)=(\xi, \phi(\xi)), \quad-1 \leq \xi \leq 1,
$$

where

$$
\phi(\xi)=\sqrt{2-x^{2}}-1
$$

under the condition on the boundary $\Gamma$ as follows:

$$
f(P)=0, \quad g(P)=1 / N(x)
$$

for all $P=\beta(x) \in \Gamma$. Numerical results for this example have been obtained from a program coded by Mathematica V4, and the standard Legendre Gauss quadrature rule with the number of integration points, NGP $=12$ has been used after the Sidi-sigmoidal transformation of order $m=5$.

For several source points, $P_{k}, k=1,2,3,4,5$, taken as

$$
P_{1}=(0, \phi(0)+0.1), \quad P_{2}=(0, \phi(0)+1), \quad P_{3}=(1.1,0)
$$

and

$$
P_{4}=(2,0), \quad P_{5}=(10,10)
$$


Table 1 and Table 2 show numerical values of the displacements, $u_{i}\left(P_{k}\right)$ and stress functions, $F\left(P_{k}\right), G\left(P_{k}\right)$ and $H\left(P_{k}\right)$, respectively, with respect to various numbers of collocation points, $N=4,8, \cdots, 24$. One can see that all of the numerical solutions for displacements and stresses converge as $N$ increases. The rate of convergence can be accelerated by using large NGP.

Table 1. Numerical results of displacements, $u_{1}$ and $u_{2}$ for each source point, $P_{i}(i=1,2,3,4,5)$ and for various number of collocation points, $N=4,8, \cdots, 24(\mathrm{NGP}=12)$.

\begin{tabular}{c|c|rr}
\hline$P_{i}$ & $N$ & \multicolumn{1}{|c}{$u_{1}\left(P_{i}\right)$} & $u_{2}\left(P_{i}\right)$ \\
\hline & 4 & $-4.99 \times 10^{-16}$ & 6.21872 \\
& 8 & $2.36 \times 10^{-16}$ & 6.19988 \\
& 12 & $-1.36 \times 10^{-16}$ & 6.20163 \\
$P_{1}$ & 16 & $-6.71 \times 10^{-16}$ & 6.20315 \\
& 20 & $-2.14 \times 10^{-16}$ & 6.20355 \\
& 24 & $9.22 \times 10^{-14}$ & 6.20361 \\
\hline \multirow{5}{*}{$P_{2}$} & 4 & $-7.35 \times 10^{-17}$ & 1.43247 \\
& 8 & $9.54 \times 10^{-17}$ & 1.46206 \\
& 12 & $3.77 \times 10^{-17}$ & 1.46741 \\
& 16 & $-3.29 \times 10^{-17}$ & 1.46920 \\
& 20 & $-3.99 \times 10^{-17}$ & 1.46985 \\
& 24 & $1.62 \times 10^{-14}$ & 1.47025 \\
\hline \multirow{5}{*}{$P_{3}$} & 4 & 0.0769053 & 0.710042 \\
& 8 & 0.0483158 & 0.563936 \\
& 12 & 0.0454997 & 0.540234 \\
& 16 & 0.0458767 & 0.532329 \\
& 20 & 0.0465580 & 0.528642 \\
& 24 & 0.0463925 & 0.526807 \\
\hline & 4 & -0.0144693 & -4.57829 \\
& 8 & -0.0161923 & -4.52149 \\
$P_{4}$ & 12 & -0.0159720 & -4.50959 \\
& 16 & -0.0154557 & -4.50592 \\
& 20 & -0.0150095 & -4.50397 \\
& 24 & -0.0150299 & -4.50280 \\
\hline & 4 & 1.97791 & -16.6662 \\
& 8 & 1.94592 & -16.3887 \\
& 12 & 1.93965 & -16.3345 \\
& 16 & 1.93769 & -16.3173
\end{tabular}




\begin{tabular}{|c|c|c|}
\hline 20 & 1.93667 & -16.3085 \\
\hline 24 & 1.93608 & -16.3034 \\
\hline
\end{tabular}

Table 2. Numerical results of stress functions, $F, G$ and $H$ for each source point, $P_{i}(i=1,2,3,4,5)$ and for various number of collocation points, $N=4,8, \cdots, 24(\mathrm{NGP}=12)$.

\begin{tabular}{|c|c|c|c|c|}
\hline Source points & $\bar{N}$ & $F\left(P_{i}\right)$ & $G\left(P_{i}\right)$ & $\bar{H}\left(P_{i}\right)$ \\
\hline \multirow{6}{*}{$P_{1}$} & 4 & -1.53470 & 0.230063 & $9.85 \times 10^{-17}$ \\
\hline & 8 & -1.50869 & 0.226362 & $-7.87 \times 10^{-17}$ \\
\hline & 12 & -1.50631 & 0.225485 & $7.25 \times 10^{-17}$ \\
\hline & 16 & -1.50562 & 0.225056 & $-1.90 \times 10^{-16}$ \\
\hline & 20 & -1.50515 & 0.224771 & $1.63 \times 10^{-17}$ \\
\hline & 24 & -1.50506 & 0.224712 & $-3.09 \times 10^{-15}$ \\
\hline \multirow{6}{*}{$P_{2}$} & 4 & -0.854899 & -0.456206 & $3.48 \times 10^{-17}$ \\
\hline & 8 & -0.844299 & -0.452825 & $-2.38 \times 10^{-17}$ \\
\hline & 12 & -0.842416 & -0.452469 & $5.367 \times 10^{-19}$ \\
\hline & 16 & -0.841793 & -0.452385 & $-1.96 \times 10^{-16}$ \\
\hline & 20 & -0.841426 & -0.452313 & $2.26 \times 10^{-17}$ \\
\hline & 24 & -0.841246 & -0.452269 & $6.97 \times 10^{-15}$ \\
\hline \multirow{6}{*}{$P_{3}$} & 4 & 0.204312 & -0.073165 & 0.382431 \\
\hline & 8 & 0.232262 & -0.096559 & 0.345530 \\
\hline & 12 & 0.238366 & -0.101830 & 0.340992 \\
\hline & 16 & 0.239724 & -0.103085 & 0.339907 \\
\hline & 20 & 0.240013 & -0.103416 & 0.339435 \\
\hline & 24 & 0.240285 & -0.103624 & 0.339038 \\
\hline \multirow{6}{*}{$P_{4}$} & 4 & 0.0458348 & -0.0301487 & 0.179745 \\
\hline & 8 & 0.0469173 & -0.0310170 & 0.176227 \\
\hline & 12 & 0.0469667 & -0.0310532 & 0.175513 \\
\hline & 16 & 0.0469383 & -0.0310351 & 0.175286 \\
\hline & 20 & 0.0469014 & -0.0310108 & 0.175174 \\
\hline & 24 & 0.0469059 & -0.0310143 & 0.175105 \\
\hline \multirow{6}{*}{$P_{5}$} & 4 & -0.0624079 & 0.000378806 & -0.0264741 \\
\hline & 8 & -0.0613682 & 0.000396734 & -0.0260292 \\
\hline & 12 & -0.0611650 & 0.000399182 & -0.0259424 \\
\hline & 16 & --0.0611008 & 0.000399539 & -0.02591 .52 \\
\hline & 20 & -0.0610675 & 0.000399439 & -0.0259012 \\
\hline & 24 & -0.0610484 & 0.000399670 & -0.0258930 \\
\hline
\end{tabular}




\section{Conclusion}

In this paper we have studied on the indirect boundary integral method, via real variables, for curved crack problems in plane elasticity. Unique solvability of the resultant system of integral equations has been proved completely. In addition, a numerical example of a circular arc crack has been given to justify the result of this paper.

As we mentioned in Section 1, one can observe that this approach may overcome the theoretical and computational difficulties in the traditional boundary integral formulation of the cracked body.

We expect further work on the convergence analysis of the approximate scheme, which assures availability and stability of the indirect boundary integral method.

\section{References}

[1] M. H. Aliabadi and D. P. Rooke, Numerical Fracture Mechanics, Kluwer Academic Publ., London, 1991.

[2] G. E. Blandford, A. R. Ingraffea, and J. A. Liggett, Two-dimensional stress intensity factor computations using the boundary element method, Intern. J. Numer. Methods Engrg. 17 (1981), 378-394.

[3] Y. Z. Chen, A survey of new integral equations in plane elasticity crack problems, Engrg. Fracture Mech. 51 (1995), 97-114.

[4] C. Constanda, The boundary integral equation method in plane elasticity, Proc. Amer. Math. Soc. 123 (1995), 3385-3396.

[5] F. Erdogan, Stress intensity factors, Trans. ASME J. Appl. Mech. 50 (1983), 992-1002.

[6] R. W. Ladner, Mathematical Theory of Dislocations and Fracture, Univ. Toronto press, Toronto, 1974.

[7] S. G. Mikhlin and S. Prössdorf, Singular Integral Operators, Springer - Verlag, Berlin, 1986.

[8] A. Portela, Dual Boundary Element Analysis of Crack Growth, Comp. Mech. Publ., Southampton, 1993.

[9] A. Portela, M. H. Aliabadi, and D. P. Rooke, The dual boundary element method; effective implementation for crack problems, Intern. J. Numer. Methods Engrg. 33 (1992), 1269-1287.

[10] U. Sur and N. J. Altiero, An alternative integral equation approach for curved and kinked cracks, Internat. J. Fracture 38 (1988), 25-41.

[11] W. L. Wendland and E. P. Stephan, A hypersingular boundary integral method for two-dimensional screen and crack problems, Arch. Ration. Mech. Anal. 112 (1990), 363-390.

[12] Y. Xu and L. M. Keer, Crack curving in anisotropic elastics solids, Engrg. Fracture Mech. 44 (1993), 63-73.

[13] B. I. Yun and S. Lee, Double layer potential scheme for dirichlet problems on smooth open arcs, Comput. Math. Appl. 37 (1999), 31-40. 
[14] P. R. Johnston and D. Elliott, Error estimation of quadrature rules for evaluating singular integrals in boundary element, Int. J. Numer. Methods Engrg. 48 (2000), 949-962.

Faculty of Mathematics, Informatics and Statistics

College of Natural Science

Kunsan National University

Kunsan 573-701, Korea

E-mail: biyun@kunsan.ac.kr 